\title{
sciendo
}

\author{
BULGARIAN ACADEMY OF SCIENCES
}

CYBERNETICS AND INFORMATION TECHNOLOGIES • Volume 18, No 4

Sofia $2018 \quad$ Print ISSN: 1311-9702; Online ISSN: 1314-4081

DOI: $10.2478 /$ cait-2018-0044

\section{Cloud-Based Test Tools: A Brief Comparative View}

\author{
Nergiz Kılınç ${ }^{1}$, Leyla Sezer ${ }^{2}$,Alok Mishra ${ }^{1}$ \\ ${ }^{1}$ Atilim University, Incek, Ankara, Turkey \\ ${ }^{2}$ İhsan Doğramacı Bilkent University, Bilkent, Ankara, Turkey \\ E-mails: nergiz.kilinc@atilim.edu.tr_loksuz@bilkent.edu.tr_alok.mishra@atilim.edu.tr
}

Abstract: The concept of virtualization has brought life to the new methods of software testing. With the help of cloud technology, testing has become much more popular because of the opportunities it provides. Cloud technologies provides everything as a service, hence the software testing is also provided as a service on cloud with the privileges of lower cost of testing, and relatively less effort. There are various cloud-based test tools focusing on different aspects of software testing such as load tests, regression tests, stress tests, performance tests, scalability tests, security tests, functional tests, browser performance tests, and latency tests. This paper investigates the cloud-based testing tools focusing on different aspects of software testing.

Keywords: Cloud Testing, Performance Testing, Load Testing, Software engineering testing strategy, Verification, Validation, Test tools.

\section{Introduction}

Cloud computing is the combination of already existig technologies that perform many functions with the virtualized resources. As the cloud model presented in Fig. 1. illustrates, the platform and applications are built upon the infrastructure provided on cloud [26]. Cloud based technologies have become very popular in the recent years due to its relatively less cost, higher performance, ease of use compared to manual testing. Although there are challenges of applying the cloud-based testing because the team must be knowledgable on how to setup a testing environment and scenarios on the cloud. Other than these challenges, it is a highly preferred method since there are almost no efforts required and it can perform better and 24/7 as it is entirely computerized. Mainly all the cloud technologies provide the organization an opportunity to invest less budget on the services they frequently use when compared to on-premise setups they install locally. The organizations only pay for the services on-demand style on cloud. Another advantage of using the cloud testing is that, once the test setting is built up on cloud, the cloud provides testing in the most consistent way, with a high performance as it is 100 percent computerized and can perform heavy tests that are not possible to test manually such as load tests or full regression tests in a very easy and effective way. This increases the demand on cloud services, 
and the more the development of cloud services increases the more there will be a need for testing the applications developed for cloud [1].

Cloud technology provides the infrastructure including hardware network bandwidth, and workload that are designated to mimic the real-world scenarios for the testing of software [3]. This enables organizations to rent the infrastructure and computerize the test environment to reduce cost and manpower, and increase performance, accuracy, and consistency.

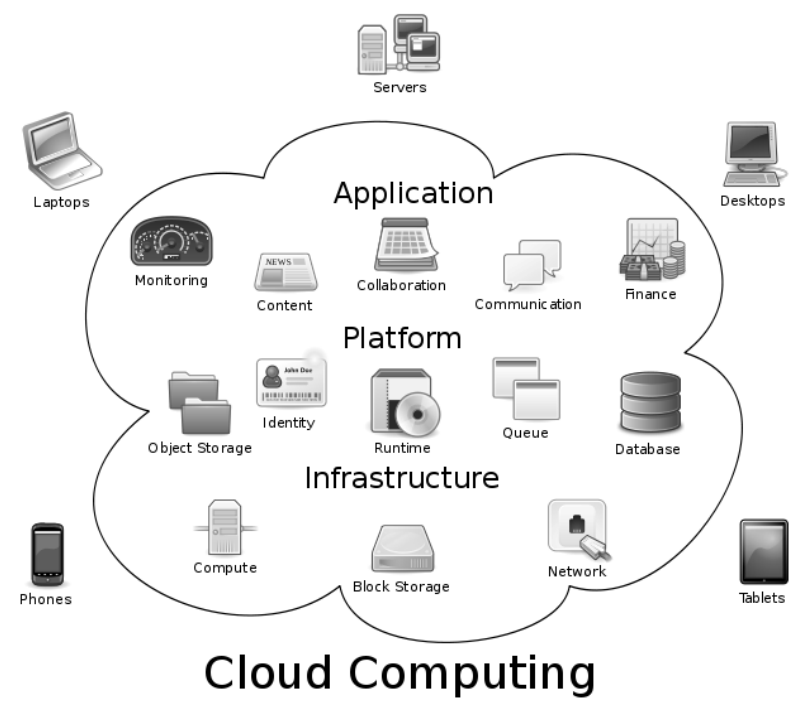

Fig. 1. Cloud computing model [26]

Cloud testing has been a revolution on the traditional licencing. The amount of on-premise licencing and installation of systems have been decreasing dramatically while the on-demand hiring of systems and infrastructure has been increasing rapidly. A study conducted in 2010 predicted that the twenty percent of IT organizations would own zero on-premise ownership. This, proves the popularity of cloud-based tools over the physically installed tools. The same is valid for the cloud-based testing too.

The following list illustrated the cloud-based test components:

- Performance and Load Testing (longevity testing): Testing the performance and stability of software under extreme workload conditions.

- Stress Testing and Recovery Testing: Testing the software strength and recovery under the extreme circumstances.

- Security Testing: Testing for ensuring that the software meets the expected security requirements.

- Functional Testing : Testing the functionality of the software.

- Browser Performance: Testing the performance of software across various browsers.

- Latency Testing: Testing the software for ensuring a consistent and acceptable response time. 
- Interoperability and Compatibility Testing: Testing software to ensure the modules in a software are interoparable to work successfully and compatible together.

Software quality has long been a critical issue for software developers [27, 28] and there is always risk involved in ensuring it [29, 30]. Additional to the listed test types, the software test teams want to consider the following aspects of quality requirement aspects during testing [2].

- Security risks

- Multiple Web browser support

- User interface issues

- User data accessibility.

The primary benefit of cloud-based testing is reduced costs for putting up, maintaining, and licensing internal testing environments [1]. Software testing on cloud reduces the time spent on setting up a test environment, dramatically, resulting in the increased efficiency of software testing. One of the greatest privileges that cloud-based testing provides is that the tests can be performed with a much greater number of clients, as it is not inter-enterprise simulation and the cloud can deal with unexpected traffic peaks better than the CPU. Normally, testing software provided for the customers is too expensive to purchase. Cloud-based testing allows customers rent the software testing tools rather than actually buying it, which is much more costefficient [25].

Additional to its benefits, there are some disadvantages of cloud testing. The cost for training the test team for having them gain the special skills for performing tests on cloud is high and requires effort, but once it is set up, the other costs, effort and time are reduced dramatically. Some costs are much harder to predict for cloudbased testing such as the costs of providing and monitoring the security. Due to the fact the infrastructure and the control belongs to the service provider, the data and information cannot be 100 percent secure, which is almost the greatest weaknesses of cloud services, hence providing that security might incur additional costs [2]. In this article, the most recent tools used for the cloud-based testing have been presented in addition to the existing cloud-based test tools. In the following part a detailed description about the test types is provided.

\section{Cloud Testing types}

There are various types of testing methods, used in the cloud. Each method is briefly explained in this section.

\subsection{Elasticity and Scalability Testing}

A software needs to be scalable and elastic. One of the major advantages of cloud is that it automatically supports elasticity such as satisfying the resource demand at the run time. On hot seasons, when the resources requirement increases, the resources should be scaled up and down automatically. Therefore, "vertical and horizontal scalability" need to be tested. Vertical scalability is the ability to replace the current resource with a more powerful resource that might satisfy the increased demand. Horizontal scalability makes use of more resources of same type in order to enhance 
the performance of the platform. Also, the needed resources have to be provisioned in a flawless way whenever the demands arise $[1,3]$.

\subsection{Functional Testing}

Software undergo a couple of test cases for functional testing. Not only Internet but also non-Internet applications can undergo functional testing on the cloud [5].

\subsection{Compatibility Testing}

Cloud environment provides option of various operating systems and the software can be tested for compatibility with different operating systems environments. It tests whether the software developed is compatible with the hardware, O.S., database or other system software or not.

\subsection{Security Testing}

When the application is on cloud, it is exposed to various security threats. One of the major security threats on cloud is the traversal vulnerability that enables an intruder to traverse from one virtual machine to another through the same hypervisor. Due to this threat, it is possible for the client to access the virtual instances of the other clients' applications. To protect the application from such a threat, penetration testing is very significant. Other security tests include the tests of client roles and their access control, "federation management, and data management" [3].

\subsection{Performance Testing}

The accuracy of the software is tested on cloud based on the performance testing. Data persistency, latency, and throughput are also the factors that affect the software performance, hence considered in the testing. Additionally, elastic load testing and multi-tenant performance testing are other performance-based factors.

For a software to be highly available and failover-proof are the most important indicators of performance. Therefore, an extensive performance testing under resilience scenarios is essential for the behavior of the software [2].

\subsection{Live Upgrade Testing}

A quality software should be able to perform the user demands even when it is performing live upgrades. It is essential that the user should not feel the effect of upgrade task, or any performance decrease during the upgrade. This test ensures the capability of software to perform with a high performance and to continue is business services during the upgrade [26].

\subsection{Stress Testing}

Stress testing is the test that ensures the capability of a software to perform effectively under severe conditions beyond the software's crashing point. It is very important for any software to perform well under extensive stress and to maintain its stability and consistency [26]. 


\subsection{Browser Performance}

A web application should be tested to understand whether it works as expected in various different browsers. There are a number of tools that performs automated browser performance tests on the cloud [4].

\subsection{Latency Testing}

It is very essential for a software to perform in a consistent way when responding to a number of actions by the user. The latency variability causes a problem as the user thinks there might be something unexpected going on in the background. Latency tests do not only ensure reducing the response time to an optimal level but also tries to enable the consistency of the latency. A software whose response varies from 0.5 seconds to 2 seconds irritates the user more than a software that responds to the demands consistently after 3 seconds, hence it is so important to perform latency tests, and there are cloud-based tools to support this quality requirement [3].

\subsection{Load Testing}

The load tests are performed by creating peak loads using simulators. The software is tested for its strength on maintaining the consistent performance without crushing in extreme amounts of loads. Creating such peak load scenarios are almost impossible to implement manually since the cost of creating such scenarios is huge. Instead of investing capital in building on-premise testing environments, cloud testing provides an affordable and scalable alternative [4] for load testing. It is very costly to install the required hardware that can handle enormous amounts of load, yet it is more affordable to hire the infrastructure necessary for the load test on cloud.

\section{Testing tools}

\subsection{Cloud Computing Testing from SPIRENT}

Spirent is known as the first methodology that is utilized to test the Performance, Availability, Security and Scalability (PASS) of the services and infrastructure provided by cloud [5].

\subsection{AppPerfect Cloud Testing}

AppPerfect is used to test the portability of web applications. With the use of this tool, the web applications can be tested on various browsers, operating systems, and hardware by using the cloud infrastructure [6].

\subsection{Automated website testing from the cloud}

Automated website testing service provides the opportunity to run automated functional tests and cross browser tests of websites by using the Selenium framework. After detecting the defects and errors, "tester" role is assigned to all users and "UserReplay" records each user's activity to fix the problems found [7]. UserReplay enables replaying the recorded user activities, which makes it easier to recreate / replicate the activity that revealed the problem occurred. 


\subsection{Zephyr Enterprise Edition}

Zephyr is provided as a "Software-as-a-Service (SaaS)" platform in order to manage the tests on cloud. The tool provides all functions of testing including "lifecyle, integration of various test tools and systems, global access, cooperation, management visibility, automation, collaboration, metrics and reporting".

\subsection{Cloud Testing with TestMaker}

TestMaker is used for running automated tests on cloud. This tool provides all the necessary equipment for automated tests including "creating the test nodes in EC2 instances, running the tests, reporting the results" [9].

\subsection{Cloud-Enabled Software Testing as a Service}

Everything on cloud is advantageous in terms of pay-per-use. Likewise, the ondemand pay-per-use tests are much more effective for the organization. When tests are run on cloud, it is provided as a "Service. Cloud infrastructure is provided to run tests on the cloud [10].

\subsection{CloudTest Platform by SOASTA}

CloudTest platform ensures that business-critical mobile and web applications will function properly and consistently even under extreme loads. SOASTA Cloudtest is a single tool that combines all the functional and performance testing methodologies for web and mobile applications. The most outstanding feature of the tool is the possibility to fast creation and editing of the most complex test cases by using "visual test creation environment" [11].

\subsection{JMeter in the Cloud}

Normally, performance and load tests are not very easy to run. However, with the help of automated tests and especially running the tests on cloud made it far more easier and possible. JMeter is known as a performance and load testing tool that runs the tests on cloud by using the cloud infrastructure. The tool enables to create and execute test cases on cloud and makes it possible to run the tests with the help of thousands of virtual users without requiring any setups [12].

\subsection{NeoLoad Cloud Testing}

NeoLoad provides load testing on the cloud provided as a service. Although it is very difficult to perform load tests manually due to the difficulty in simulating the large loads on the applications, it is very simplified on cloud. Neoload load testing tooş enables load generation from cloud services into the testing operations [13].

\subsection{Parasoft SOAtest}

Parasoft SOAtest enables automated "web application testing, message/protocol testing, cloud testing and security testing". Using the Parasoft SOAtest and Parasoft Load Test together enables "secure, reliable, and compliant" business processes.This tool is used to detect application layer defects at the early stages of software development lifecycle [14]. 


\subsection{Cloud-Based Cross-Browser Testing: Sauce Labs}

Sauce Labs tools are created to provide browser testing for quality assurance. The created application is tested on various browsers by using a Virtual Machine (VM) that runs on cloud. In order to run the test, the tool requires a URL to test, select the operating system and browser and the VM [15].

\subsection{Skytap}

Skytap is an automation solution runs on cloud for the companies and software dealers to test and improve the developed software based on the quality standards [16].

\subsection{Software Testing In The Cloud (STITC)}

The primary objective of STITC is examining methods for migrating legacy testing assets to the cloud [17]. The Software Testing In The Cloud (STITC) series of events follows the principle of utilizing the services and infrastructure provided on cloud to run the tests. Everything on cloud is provided as a service, hence software testing is also provided as a service. There are various categories of testing provided in the cloud services such as functional testing, Model-Based Testing (MBTIC), performance testing as a service, security testing as a service, and a number of others [22].

See Appendix to view at a glance for the tools with their testing methodologies.

\subsection{Cloud Unit Testing Tool (CUT)}

The speed of unit tests can be increased significantly by performing the tests on cloud services, yet it is a challenging task to proceed, and manage the tests manually on the cloud, as it requires a team with special expertise to perform the test. Training the team and carrying the test on the distributed systems therefore can take a lot of time and can be costly. The Cloud Unit Testing (CUT) tool is developed to perform the unit tests on distributed execution environments automatically. CUT allows resource allocation, test clustering and distribution for developers [18].

\subsection{LoadStorm}

Load testing is considered as one of the most challenging tests to apply due to the vast utilization of resources and human workforce. It is highly mentioned in the literature that it is almost impossible to implement the load tests manually. The tool called LoadStorm is developed for transferring the entire load testing process into cloud. The tool provides web and mobile interfaces for applying various load test scenarios that are customizable in a cost effective and easier way [19].

\subsection{App Thwack}

App Thwack tool provides a cloud-based test simulator for the mobile web applications. The tool provides an API that enables the apps to be tested on the actual client site rather than the developer site. It is very crucial to provide test reports appropriate for the standards and the requirements of the firms; hence, this tool eases the production of reports [20]. 


\subsection{Xamarin}

The main concern of the tool is to provide an ease of testing process for the user interface acceptance. The tool helps to run the tests on thousands of mobile devices and provides highest resolution screen shots of the information required to be documented such as the CPU and memory consumption [21].

\subsection{SDNort}

Chi and his colleagues developed a software defined network-testing framework using Openflow that is virtualized and deployed on the cloud as a service. The main functionality of the tool is to create network throughput traffic to test the network performance and produce reports including statistics. The fact that SDNort does not produce the traffic through CPU computation enhances the performance of testing, as it is highly lightweight and is not dependent on the CPU performance [23].

\subsection{BRACE: Cloud-Based Software Reliability Assurance}

Okumoto and his colleagues developed a tool that works on the cloud for testing providing software reliability. The tool has various functionalities including the software reliability modeling, testing, and defect analysis. The tool provides a realtime statistic of the total number of defects and errors on the developing software, and generates statistical reports on the conclusion [24].

\section{Testing Challenges}

\subsection{Constructing Environment For On Demand Testing}

For an on-demand testing service, it is required to ensure the steps to be taken to create a testing environment systematic or automatic ones. Even though the present technologies in cloud support instinctive delivery of needed computing resources for every Software as a Service (SaaS) or for an application in the cloud, for establishing the required test domain in a cloud there are no carrying solutions to assist engineers using a beneficial way [4].

\subsection{Testing Security Measures}

In present software testing society security testing is been converting to a subtle topic with lot of open queries. Meanwhile security turn into a most important requirement within the clouds and security services converted into a compulsory measure in present Software as a Service (SaaS) and cloud technology, the problems and challenges in security validation and quality assurance for SaaS and clouds need to be deal by the engineers [4].

\subsection{Integration Testing}

Even though there are research related with software integration testing problems and schemes, still there is paucity in the real engineering system area. The main purpose are the software and mechanisms are created without allowing technology and solution to assist coordinated software integration. In a cloud organization, software developers required to be deal with combination of various SaaS and applications 
inside and outside clouds in a black-box sight depending on the offered APIs and connectivity protocols [4].

\subsection{Regression Testing}

The regression testing problems and challenges induced by software modifications and bug-fixing must be addressed the by on-demand software validation in clouds. Yet, maximum current exploration in software regression testing grants greater deliberation to re-test a particular software version in a preconfigured test environment. The multi-tenancy characteristic of clouds might origin the concern to put on the present explored work in cloud testing, notably for on-demand software regression testing service each and every time software is modified [4].

\section{Similarities and differences of the tools}

From cloud testing tools observations, it seems most of the testing tools have general similar attributes. But some of them are more useful than others. In this section, similarities, and differences of each tool has been discussed. SPIRENT, Sogeti, SOASTA, Parasoft SOATest and STITC have emphasize Scalability, Security and Performance testing. AppPerfect, UserReplay, SauceLabs, SOASTA, Parasoft SOATest, STITC are the tools used for Web Applications Testing. In addition to this, Zephr, TestMaker, UserPlay, JMeter, Skytap, STITC adopt the automated testing, which is one of the most significant testing type in software engineering. There are common and unique features of each cloud based testing tool, which are represented in Table 1 in detail manner.

Stress tests commonly put a greater priority on robustness, availability, and error handling under excessive load, than on what would be considered correct behavior under normal circumstances. SOASTA and Zepher highly uses the stress testing inside their tools.

Compatibility testing is another significant testing method for the SE. It tests whether the application or the software product built is adaptable with the hardware, operating system, database or other system software. SPIRENT, Parasoft SOAtest and STITC point out the compatibility testing.

Another term is Latency, which is the delay from input into a system to desired outcome; the term is understood slightly differently in various contexts and latency issues also vary from one system to another. SOASTA, JMeter, NeoLoad notice and uses Latency, load and performance testing.

In general, all of the testing tools make Performance and Functional testing strategies. Because software engineering management, firstly seeks the performance and functional testing. So, all of them implements these tests first. However, nowadays the need of security, stress and automated testing strategies are rising in SE projects.

From this view, to sum up, tools which uses browser testing tools are more preferred than other tools. These are SOASTA, AppPerfect, UserPlay, Sauce Labs, Parasoft SOATest and STITC. As there are lots of web applications, client/server applications so, people, companies, organizations preferred these ones. But later on, 
it is clear that stress, security and automated testing needs will be increase, therefore these capabilities will be change. From this perspective, Zephr, JMeter, Skytap and STITC are better tools. Also, I want to recommend SOASTA can include automated testing to its structure because it highly highlight the other technics also attach automated testing to compete the future plan and also to increase it benefits.

\section{Conclusion}

To conclude, in upcoming years cloud computing is emerging in IT industry the real opportunity to cut cost of test environment in public private and hybrid environments or community clouds lies in both their management and maintenance before deploying the cloud its essential that cloud application is thoroughly tested. In this paper, different cloud testing techniques and commercial tools available in the market have been discussed. This paper identified some of the challenges and also similarities and differences through the analysis. The findings have been summarized below:

- Most of the testing tools have similar attributes.

- SPIRENT, Sogeti, SOASTA, Parasoft SOATest and STITC have emphasize Scalability, Security and Performance testing.

- AppPerfect, UserReplay, SauceLabs, SOASTA, Parasoft SOATest, STITC, and AppThwack are the tools which used for Web Applications Testing.

- Cloud Unişt Testing Tool (CUT) performs unit tests on distributed execution environments, allows resource allocation, test clustering and distribution.

- Zephr, TestMaker, UserPlay, JMeter, Skytap, STITC uses the automated testing.

- Stress tests commonly put a greater emphasis on robustness, availability, and error handling under a heavy load, than on what would be considered correct behavior under normal circumstances. SOASTA and Zepher highly uses the stress testing inside their tools.

- SPIRENT, Parasoft SOAtest and STITC emphasize the compatibility testing.

- SOASTA, JMeter, NeoLoad, LoadStorm, Xamarin, SDNort notice and uses Latency, load and performance testing.

- BRACE performs software reliability modeling, testing and defect analysis. The limitations of this study are that there is limited research in the literature about the emerging cloud-based test tools. Therefore, the tools are discovered through the websites as well as the literature. This study will be extended for more cloud-based test tools. Because there are limited research in the literature about the subject matter, the following study may contribute to the literature.

\section{References}

1. R i u n g u-Kalli o s a a r i, L., O. T a i p a l e, K. S m o l an d e r (n. d.). 27 September 2012. https://www.infoq.com/articles/testing-in-the-cloud-exploring-the-practice

2. Cloud Testing (n. d.). https://www.techopedia.com/definition/26540/cloud-testing 
3. N a chi y a p p a n a, S., S. Ju st u s b. Cloud Testing Tools and Its Challenges: A Comparative Study. - In: 2nd International Symposium on Big Data and Cloud Computing, 2015, (ISBCC'15).

4. A b h a n g, R. D., B. B. G i t e. Testing Methods and Tools in a Cloud Computing Environment. International Journal of Engineering Research \& Technology (IJERT) IJERT Vol. 3, November 2014, Issue 11.

5. Automated Testing with Sprint (n.d). http://www.spirent.com/NetworksandApplications/Cloud_Computing.aspx

6. Performance and Functional Testing (n.d.) http://appperfect.com/products/cloudcomputing/cloud-testing.html

7. Customer Experience Analytics (n.d.). https://www.userreplay.com/

8. Real Time Testing with Agile Tools (n.d.). http://www.getzephyr.com/

9. Cloud Testing (n.d.). http://www.pushtotest.com/cloud-testing.html

10. Cloud Enabled Software Testing as a Service (n.d.). http://www.sogeti.com/looking-forsolutions/Services/Infrastructure-Management/Cloud-Computing/Cloud-Enabled-SoftwareTesting-as-a-Service/

11. Cloud Test (n.d.) http://www.soasta.com/cloudtest/

12. Cloud Testing (n.d.). http://www.cloud-intelligence.com/cloud/testing

13. Neolan Cloud Testing (n.d.). http://www.neotys.com/product/neoload-cloudtesting.html

14. SOATEST (n.d.). http://www.parasoft.com/jsp/products/soatest.jsp

15. Cloud Based Browser Testing (n.d.). http://konigi.com/tools/submissions/sauce-labscloud-based-cross-browser-testing

16. Skytab Cloud Testing (n.d.). http://www.skytap.com/skytap-cloud/

17. STITC: Software Testing in the Cloud (n.d.). http://stitc.org/

18. G a m b i, A., S. K a p p l e r, J. L a m p e 1, A. Z e 11 e r. CUT: Automatic Unit Testing in the Cloud. - In Proc. of 26th ACM SIGSOFT International Symposium on Software Testing and Analysis (ISSTA'17), 2017, ACM, New York, NY, USA, 364-367.

19. How to Load Test in Six Steps With LoadStorm (n.d.). https://loadstorm.com/

20. Testing by Appthwack (n.d.) https://appthwack.com/

21. Introduction to Xamarin Test Cloud (n.d.). https://developer.xamarin.com/testcloud/

22. Bertolino, A., L. Nautiyal, Preeti. Annotated Buzzwords and Key References for Software Testing in the Cloud. - In: 2017 International Conference on Computing, Communication and Automation (ICCCA'17), Greater Noida, India, 2017, pp. 893-900.

23. Ch i, P. W., M. H. W a n g, C. W. L i n, J. W. G u o, C. L. L e i, N. F. H u a n g. SDNort: A Software Defined Network Testing Framework Using Openflow. - In: 2016 11th Asia Joint Conference on Information Security (AsiaJCIS'16), Fukuoka, 2016, pp. 100-107.

24. Okumoto, K., A. A sthana, R. Miju mbi. BRACE: Cloud-Based Software Reliability Assurance. - In: 2017 IEEE International Symposium on Software Reliability Engineering Workshops (ISSREW'17), Toulouse, 2017, pp. 57-60.

25. J u n, W., F. M e n g. Software Testing Based on Cloud Computing. - In: 2011 International Conference on Internet Computing and Information Services, Hong Kong, 2011, pp. 176-178.

26. N a c h i y a p p a n, S., S. J u s t u s. Cloud Testing Tools and Its Challenges: A Comparative Study. - In: 2nd International Symposium on Big Data and Cloud Computing (ISBCC'15), Elsevier, 2015, pp. 482-489. 
27. Mishra, D., A. Mishra. Simplified Software Inspection Process in Compliance with International Standards. - Computer Standards \& Interfaces, Vol. 31, 2009, No 4, pp. 763-771.

28. Mishra, A., D. Mishra. Software Quality Assurance Models in Small and Medium Organisations: A Comparison. - International Journal of Information Technology and Management, Vol. 5, 2006, No 1, pp. 4-20.

29. M i s h r a, D., A. M i s h r a. A Review of Non-Technical Issues in Global Software Development. - International Journal of Computer Applications in Technology, Vol. 40, 2011, No 3, pp. 216-224.

30. Y u, L., A. M i s h r a. Risk Analysis of Global Software Development and Proposed Solutions. Journal Automatika, Vol. 51, 2010, No 1, pp. 89-98.

\section{Appendix}

Table 1. Cloud Test tool features

\begin{tabular}{|l|c|c|c|c|c|c|c|c|c|c|}
\hline $\begin{array}{c}\text { Tool names } \\
\text { and Testing } \\
\text { types }\end{array}$ & $\begin{array}{c}\text { Scala- } \\
\text { bility }\end{array}$ & $\begin{array}{c}\text { Secu- } \\
\text { rity }\end{array}$ & $\begin{array}{c}\text { Func- } \\
\text { tional }\end{array}$ & $\begin{array}{c}\text { Perfor- } \\
\text { mance }\end{array}$ & $\begin{array}{c}\text { Live } \\
\text { upgrade }\end{array}$ & Stress & $\begin{array}{c}\text { Compa- } \\
\text { tibility }\end{array}$ & Browser & Latency & $\begin{array}{c}\text { Automated } \\
\text { and } \\
\text { reporting }\end{array}$ \\
\hline SPIRENT & $\checkmark$ & $\checkmark$ & & $\checkmark$ & & & $\checkmark$ & & & \\
\hline AppPerfect & & & $\checkmark$ & & $\checkmark$ & & & $\checkmark$ & & \\
\hline UserReplay & & & $\checkmark$ & $\checkmark$ & & & & $\checkmark$ & & $\checkmark$ \\
\hline Zephyr & $\checkmark$ & & & $\checkmark$ & & $\checkmark$ & & & & $\checkmark$ \\
\hline TestMaker & & & & & & & & & & $\checkmark$ \\
\hline Sogeti & $\checkmark$ & & $\checkmark$ & $\checkmark$ & & & & & & \\
\hline SOASTA & $\checkmark$ & $\checkmark$ & $\checkmark$ & $\checkmark$ & & $\checkmark$ & & $\checkmark$ & $\checkmark$ & \\
\hline JMeter & $\checkmark$ & & & $\checkmark$ & & & & & $\checkmark$ & $\checkmark$ \\
\hline NeoLoad & & & $\checkmark$ & $\checkmark$ & & & & & $\checkmark$ & \\
\hline $\begin{array}{l}\text { Parasoft } \\
\text { SOAtest }\end{array}$ & $\checkmark$ & & $\checkmark$ & $\checkmark$ & & & $\checkmark$ & $\checkmark$ & & \\
\hline Sauce Labs & & & $\checkmark$ & $\checkmark$ & & & & $\checkmark$ & & \\
\hline Skytap & & & & & & & & & & $\checkmark$ \\
\hline STITC & $\checkmark$ & $\checkmark$ & & $\checkmark$ & & & $\checkmark$ & $\checkmark$ & & $\checkmark$ \\
\hline CUT & $\checkmark$ & & $\checkmark$ & & $\checkmark$ & & $\checkmark$ & $\checkmark$ & & $\checkmark$ \\
\hline LoadStorm & $\checkmark$ & & & $\checkmark$ & $\checkmark$ & & & $\checkmark$ & & $\checkmark$ \\
\hline $\begin{array}{l}\text { App } \\
\text { Thwack }\end{array}$ & $\checkmark$ & & $\checkmark$ & $\checkmark$ & & & $\checkmark$ & & & $\checkmark$ \\
\hline Xamarin & & & & $\checkmark$ & & & & & $\checkmark$ & $\checkmark$ \\
\hline SDNort & & $\checkmark$ & & $\checkmark$ & $\checkmark$ & $\checkmark$ & & & & $\checkmark$ \\
\hline BRACE & & & $\checkmark$ & & $\checkmark$ & & & & & $\checkmark$ \\
\hline
\end{tabular}

Received 16.06.2018; Accepted 21.09.2018 